\title{
Mirror fibrations and root stacks of weighted projective spaces
}

\author{
Ignacio de Gregorio and Étienne Mann
}

18 October, 2007

\begin{abstract}
We show that the orbifold Chow ring of a root stack over a well-formed weighted projective space can be naturally seen as the Jacobian algebra of a function on a singular variety.
\end{abstract}

\section{Introduction}

According to A. Givental (Giv98] ) and S. Barannikov ([Bar00]), the mirror partner of the projective space $\mathbb{P}^{n}$ is the function $f_{1}=x_{0}+\cdots+x_{n}$ on the torus defined by $x_{0} \cdots x_{n}=1$. This mirror theorem states an isomorphism between the Frobenius manifolds obtained by unfolding $f_{1}$ and the quantum cohomology of $\mathbb{P}^{n}$. To explain the motivation behind this article let us think of $f=x_{0}+\cdots+x_{n}$ as defined on the fibration $\pi: \mathbb{A}^{n+1} \rightarrow \mathbb{A}^{1}$ given by $\pi\left(x_{0}, \ldots, x_{n}\right)=x_{0} \cdots x_{n}$. From this point of view, it is natural to consider deformations of $f$ as unfoldings $F(x, t): \mathbb{A}^{n+1} \times \mathbb{A}^{k} \rightarrow \mathbb{C}$ satisfying $F(x, 0)=f(x)$, together with the equivalence relation induced by commutative diagrams

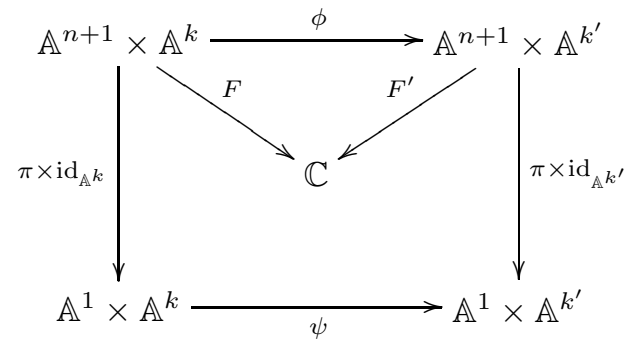

Standard techniques (e.g. dG06) show that, at least at the level of germs, the tangent space to the corresponding deformation functor, denoted by $T_{f / \pi}^{1}$, is given by the algebra

$$
T_{f / \pi}^{1}=\frac{\mathbb{C}\left[x_{0}, \ldots, x_{n}\right]}{(\pi)+\Theta_{\pi}(f)}
$$

where $\Theta_{\pi}$ denotes the vector fields on $\mathbb{A}^{n+1}$ tangent to all the fibres of $\pi$. We will refer to this algebra as the Jacobian algebra of $f$ at the 0 -fibre of $\pi$. In the case of the mirror of $\mathbb{P}^{n}$, it is readily seen that $\Theta_{\pi}$ is freely generated by the vector fields

$$
x_{i} \partial_{x_{i}}-x_{i+1} \partial_{x_{i+1}}, i=0, \ldots, n-1 .
$$

Therefore we have

$$
T_{f / \pi}^{1}=\frac{\mathbb{C}[x]}{\left(x^{n+1}\right)} \simeq H^{*}\left(\mathbb{P}^{n} ; \mathbb{C}\right)
$$

It is in this sense that seems natural to us to call the pair $(f, \pi)$ the mirror fibration of $\mathbb{P}^{n}$.

Let $p_{0}, \ldots, p_{n}$ be integer greater or equal than one. In the case of the weighted projective space $\mathbb{P}\left(p_{0}, \ldots, p_{n}\right)$, it has also been recently proved that the restriction of the function $f=x_{0}+\cdots+x_{n}$ to the torus $x_{0}^{p_{0}} \ldots x_{n}^{p_{n}}=1$ is the mirror partner of $\mathbb{P}\left(p_{0}, \ldots, p_{n}\right)$. This 
result appears as a conjecture in Man06 and it follows after the calculation of the small quantum orbifold cohomology of $\mathbb{P}\left(p_{0}, \ldots, p_{n}\right)$ by T. Coates et al. ([CCLT06]).

In this note, we construct a mirror fibration, in the sense explained above, for a toric orbifold whose coarse moduli space is a well-formed weighted projective space. In order to state our main result, we first introduce some notations.

A sequence of weights $\mathbf{p}:=\left(p_{0}, \ldots, p_{n}\right) \in\left(\mathbb{N}_{>0}\right)^{n+1}$ is called well-formed if for any $i \in\{0, \ldots, n\}$ we have $\operatorname{gcd}\left(p_{0}, \ldots, \widehat{p}_{i}, \ldots, p_{n}\right)=1$. A weighted projective space $\mathbb{P}(\mathbf{p})$ is called well-formed if its weights are well-formed.

As explained in Section 5 of [FMN07, a toric orbifold whose coarse moduli space is a well-formed weighted projective space $\mathbb{P}(\mathbf{p})$ can be encoded by a $(n+1)$-tuple $\mathbf{w}:=$ $\left(w_{0}, \ldots, w_{n}\right) \in\left(\mathbb{N}_{>0}\right)^{n+1}$ which are the multiplicities of the toric divisors. Such a toric orbifold is denoted by $\mathcal{X}(\mathbf{w}, \mathbf{p})$.

Now we state our main theorem.

Theorem 1.1. Let $\mathbf{p}:=\left(p_{0}, \ldots, p_{n}\right) \in\left(\mathbb{N}_{>0}\right)^{n+1}$ be a sequence of well-formed weights. Let $\mathbf{w}:=\left(w_{0}, \ldots, w_{n}\right) \in\left(\mathbb{N}_{>0}\right)^{n+1}$. There exists a fibration $\pi_{\mathbf{p}}: \mathcal{Y}(\mathbf{p}) \rightarrow \mathcal{C}(\mathbf{p})$ over a rational curve together with a function $f_{\mathbf{w}}: \mathcal{Y}(\mathbf{p}) \rightarrow \mathbb{C}$ such that

(a) the generic fibre $\pi_{\mathbf{p}}^{-1}(t), t \neq 0$ is isomorphic to the torus $x_{0}^{p_{0}} \ldots x_{n}^{p_{n}}=1$ and $f_{\mathbf{w}}$ is given by $x_{0}^{w_{0}}+\cdots+x_{n}^{w_{n}}$ under this isomorphism;

(b) we have a ring isomorphism

$$
T_{f_{\mathbf{w}} / \pi_{\mathbf{p}}}^{1} \simeq A_{\text {orb }}^{*}(\mathcal{X}(\mathbf{w}, \mathbf{p}) ; \mathbb{C})
$$

where the right-hand side denotes the orbifold Chow ring of $\mathcal{X}(\mathbf{w}, \mathbf{p})$.

The definition of the orbifold cohomology can be found in CR04 or in AGV02. To prove the theorem above, we only use the fact that the $\operatorname{gcd} \mathbf{p}=1$ and not that the $p_{i}$ 's are well-formed. Nevertheless, it is not a more general case as it is explain in Proposition 4.1. We put this assumption in the theorem for the following reason. As stated, this theorem highlights the role of the fibration and the function with respect to the toric orbifold $\mathcal{X}(\mathbf{w}, \mathbf{p})$. Indeed, the coarse moduli space is encoded by the fibration $\pi_{\mathbf{p}}$ whereas the root of toric divisors are encoded by the function $f_{\mathrm{w}}$. It would be interesting to see how to encode the essentially gerbe structure appeared in Section 6 of FMN07 for the mirror fibration.

The reader wanting to know what $\pi_{\mathbf{p}}$ and $f_{\mathbf{w}}$ look like, might wish to have a look at the last section of this note before reading any further.

Convention 1.2. An orbifold is a smooth DM stack of finite type over $\mathbb{C}$ with trivial generic stabiliser.

\section{Orbifold Chow ring of smooth toric DM stacks}

First we recall some general facts on smooth toric DM stacks. According to BCS05] a stacky fan denoted by $\boldsymbol{\Sigma}$ is a triple $(N, \Sigma, \beta)$ where $N$ is a finitely generated abelian group $N, \Sigma$ is a simplicial fan in $N_{\mathbb{Q}}:=N \otimes_{\mathbb{Z}} \mathbb{Q}$ with $n+1$ rays and $\beta: \mathbb{Z}^{n+1} \rightarrow N$ is a group homomorphism such that the image of the standard basis, denoted by $\left(\mathbf{e}_{0}, \ldots, \mathbf{e}_{n}\right)$ of $\mathbb{Z}^{n+1}$ generates the rays of $\Sigma$. To this combinatorial data, one can associate a smooth DM stack denoted by $\mathcal{X}(\boldsymbol{\Sigma})$. We will not use explicitly this construction so we refer to BCS05] for it.

Denote by $\mathbb{Q}[N]^{\Sigma}$ denotes the deformed group ring, that is, the underlying vector space is simply $\mathbb{Q}[N]$ but the multiplication has been deformed according to the rule

$$
y^{c_{1}} \cdot y^{c_{2}}= \begin{cases}y^{c_{1}+c_{1}} & \text { if there exists a cone } \sigma \in \Sigma \text { such that } c_{1}, c_{2} \in \sigma \\ 0 & \text { otherwise. }\end{cases}
$$

Let $\theta \in N^{\vee}:=\operatorname{Hom}_{\mathbb{Z}}(N, \mathbb{Z})$. We define the $\mathbb{Q}[N]^{\Sigma^{-} \text {-linear morphism }}$

$$
\begin{aligned}
\xi_{\theta}: \mathbb{Q}[N]^{\Sigma} & \longrightarrow \mathbb{Q}[N]^{\Sigma} \\
y^{c} & \longmapsto \theta(c) y^{c}
\end{aligned}
$$

One can prove easily the following lemma. 
Lemma 2.1. For any $\theta \in N^{\vee}$, the linear morphism $\xi_{\theta}$ is a derivation of $\mathbb{Q}[N]^{\Sigma}$.

We finish our recall by stating the main result of [BCS05]. The ring $A_{\text {orb }}^{\star}(\mathcal{X}(\boldsymbol{\Sigma}))$ is isomorphic to

$$
\frac{\mathbb{Q}[N]^{\Sigma}}{\left\langle\xi_{\theta}\left(\sum_{i=0}^{n} y^{\beta\left(\mathbf{e}_{i}\right)}\right): \theta \in N^{\vee}\right\rangle} .
$$

Remark 2.2. From Lemma 2.1 and the description of the orbifold Chow ring above, it seems natural to see $\mathbb{Q}[N]^{\Sigma}$ as the fibration and $\sum_{i=0}^{n} y^{\beta\left(\mathbf{e}_{i}\right)}$ as a function. We will explicit this in the next section on our examples.

Let $\mathbf{p}:=\left(p_{0}, \ldots, p_{n}\right) \in\left(\mathbb{N}_{>0}\right)^{n+1}$ be a sequence of well-formed weights. Let $\mathbf{w}:=$ $\left(w_{0}, \ldots, w_{n}\right) \in\left(\mathbb{N}_{>0}\right)^{n+1}$. Now, we describe the stacky fan of the toric orbifold $\mathcal{X}(\mathbf{w}, \mathbf{p})$.

The finitely abelian group $N$ is $\mathbb{Z}^{n+1} /\left\langle\sum_{i=0}^{n} p_{i} \mathbf{e}_{i}\right\rangle$. As the $p_{i}$ are coprime, the abelian group $N$ is free of rank $n$. The vector space $N \otimes_{\mathbb{Z}} \mathbb{Q}$ comes equipped with a natural simplicial fan $\Sigma$ given by the projections of the non-negative coordinate subspaces in $\mathbb{Z}^{n+1} \otimes_{\mathbb{Z}} \mathbb{Q}$. More precisely, for $k \in\{0, \ldots, n\}$, the set of $k$-dimensional cones of $\Sigma$ is given by

$$
\sigma_{J}:=\left\{\sum_{j \in J} \lambda_{j}\left[\mathbf{e}_{j}\right]: \lambda_{j} \geq 0 \in \mathbb{Q}\right\}
$$

where $J \subset\{0, \ldots, n\}$ runs through all the subsets with $k$ elements. In order to define the homomorphism $\beta$, we choose a point $w_{i} \mathbf{e}_{i} \in \mathbb{Z}^{n+1}, w_{i}>0$. If $W$ denotes the diagonal matrix $\left(w_{0}, \ldots, w_{n}\right)$, we define $\beta$ as the composite

$$
\beta: \mathbb{Z}^{n+1} \stackrel{W}{\longrightarrow} \mathbb{Z}^{n+1} \rightarrow N .
$$

We denote by $\mathcal{X}(\mathbf{w}, \mathbf{p})$ the smooth DM stack associated to the stacky fan $(N, \Sigma, \beta)$.

To have a more geometrical grasp on $\mathcal{X}(\mathbf{w}, \mathbf{p})$, we use the bottom-up construction and Section 7 of [FMN07. We deduce that the coarse moduli space of $\mathcal{X}(\mathbf{w}, \mathbf{p})$ is $\mathcal{X}(\mathbf{1}, \mathbf{p})$ where all the $w_{i}$ 's are 1 . It is a straightforward computation to see that $\mathcal{X}(\mathbf{1}, \mathbf{p})$ is the well-formed weighted projective space $\mathbb{P}(\mathbf{p})$. Denote by $\mathcal{T}:=\left[\left(\mathbb{C}^{*}\right)^{n+1} / \mathbb{C}^{*}\right]$ where the action of $\mathbb{C}^{*}$ on $\left(\mathbb{C}^{*}\right)^{n+1}$ is given by :

$$
\lambda \cdot\left(x_{0}, \ldots, x_{n}\right):=\left(\lambda^{p_{0}} x_{0}, \ldots, \lambda^{p_{n}} x_{n}\right) .
$$

The $\mathcal{X}(\mathbf{1}, \mathbf{p}) \backslash \mathcal{T}$ is a simple normal crossing divisor with irreducible components denoted by $\mathcal{D}_{i}$. Denote $\mathcal{D}:=\left(\mathcal{D}_{0}, \ldots, \mathcal{D}_{n}\right)$. The $\mathbf{w}$-th root stack of $(\mathcal{X}(\mathbf{1}, \mathbf{p}), \mathcal{D})$ is the fibber product

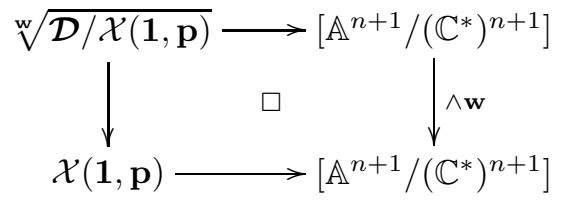

where the stack morphism $\wedge \mathbf{w}:\left[\mathbb{A}^{n+1} /\left(\mathbb{C}^{*}\right)^{n+1}\right] \rightarrow\left[\mathbb{A}^{n+1} /\left(\mathbb{C}^{*}\right)^{n+1}\right]$ is defined by sending $x_{i} \mapsto x_{i}^{w_{i}}$ and $\lambda_{i} \mapsto \lambda_{i}^{w_{i}}$ where $x_{i}$ (resp. $\lambda_{i}$ ) is the coordinates of $\mathbb{A}^{n+1}\left(\right.$ resp. $\left.\left(\mathbb{C}^{*}\right)^{n+1}\right)$. Section 7 of [FMN07, we deduce that $\mathcal{X}(\mathbf{w}, \mathbf{p})$ is isomorphic to $\sqrt[w]{\mathcal{D} / \mathcal{X}(\mathbf{1}, \mathbf{p})}$.

Remark 2.3. Let $a \in \mathbb{N}$ such that $\operatorname{gcd}\left(a, p_{n}\right)=1$. Then we have that

$$
\mathcal{X}\left(\left(w_{0}, \ldots, w_{n}\right),\left(a p_{0}, \ldots, a p_{n-1}, p_{n}\right)\right) \simeq \mathcal{X}\left(\left(w_{0}, \ldots, a w_{n}\right),\left(p_{0}, \ldots, p_{n}\right)\right)
$$

\section{Orbifold Chow ring as Jacobian algebra}

In this section we construct the fibration with the properties described in the introduction.

Overview 3.1. Looking at the orbifold Chow ring in $(\mathbb{8})$, we will see $\mathbb{Q}[N]^{\Sigma}$ as a ring defining the fibration $\pi_{\mathbf{p}}: \mathcal{Y}(\mathbf{p}) \rightarrow \mathcal{C}(\mathbf{p})$ and $\sum_{i=0}^{n} y^{\beta\left(\mathbf{e}_{i}\right)}$ as the function $f_{\mathbf{w}}: \mathcal{Y}(\mathbf{p}) \rightarrow \mathbb{Q}$. Using this idea, we will see the ring $A_{\text {orb }}^{*}(\mathcal{X}(\mathbf{w}, \mathbf{p}))$ as a Jacobian algebra. 
We first wish to express $\mathbb{Q}[N]^{\Sigma}$ as the quotient of a polynomial algebra by an ideal. In order to do so, we define

$$
\begin{aligned}
\widetilde{\alpha}: \mathbb{Z}^{n+1} & \longrightarrow\left(\mathbb{Q}_{\geq 0}\right)^{n+1} \\
\mathbf{a}:=\left(a_{0}, \ldots, a_{n}\right) & \longmapsto \mathbf{a}-\gamma(\mathbf{a}) \mathbf{p}
\end{aligned}
$$

where $\gamma(\mathbf{a}):=\min \left\{\frac{a_{i}}{p_{i}}: i=0, \ldots, n\right\}$.

The map $\widetilde{\alpha}$ admits the following interpretation: $\widetilde{\alpha}(\mathbf{a})$ is the point of intersection of the line $\mathbf{a}+\lambda \mathbf{p}$ with the set $\left\{\left(x_{0}, \ldots, x_{n}\right) \in\left(\mathbb{Q}_{\geq 0}\right)^{n+1}\right.$ such that $\left.x_{0} \cdots x_{n}=0\right\}$. It thus descends to a map $\alpha: N \rightarrow(\mathbb{Q} \geq 0)^{n+1}$. Denote also by a the class of $\mathbf{a}$ in $N$. We also see from this interpretation that

$$
\alpha\left(\mathbf{a}+\mathbf{a}^{\prime}\right)=\alpha\left(\alpha(\mathbf{a})+\alpha\left(\mathbf{a}^{\prime}\right)\right)
$$

and if $\sigma_{J} \subset N_{\mathbb{Q}}$ denotes the cone of $\Sigma$ defined in (9) then

$$
\alpha\left(\sigma_{J}\right) \subset\left\{\left(x_{0}, \ldots, x_{n}\right) \in(\mathbb{Q} \geq 0)^{n+1} \text { such that } x_{i}=0 \text { for } i \notin J\right\} .
$$

Notice that $\mathbf{a}, \mathbf{a}^{\prime} \in N$ are not in the same cone if and only if for any $i \in\{0, \ldots, n\}, a_{i}+a_{i}^{\prime}>0$. It follows that $\alpha\left(\mathbf{a}+\mathbf{a}^{\prime}\right)=\alpha(\mathbf{a})+\alpha\left(\mathbf{a}^{\prime}\right)$ if and only if there exists $\sigma \in \Sigma$ with $\mathbf{a}, \mathbf{a}^{\prime} \in \sigma$. Denote by $S \subset(\mathbb{Q} \geq 0)^{n+1}$ the semigroup (with unity) generated by $\alpha(N)$. Denote by $\mathbb{Q}[S]$ the algebra generated by $S$. As usual, we denote by $z_{i}$ the element $\alpha\left(\mathbf{e}_{i}\right)$ in $\mathbb{Q}[S]$ and we write $\mathbf{z}^{\mathbf{b}}:=z_{0}^{b_{0}} \ldots z_{n}^{b_{n}}$ for the element $\mathbf{b} \in \mathbb{Q}[S]$. The definition of $\mathbb{Q}[N]^{\Sigma}$ and the above discussion imply that

$$
\mathbb{Q}[N]^{\Sigma} \simeq \frac{\mathbb{Q}[S]}{\left(\left\{\mathbf{z}^{\mathbf{b}}: \mathbf{b}=\left(b_{0}, \ldots, b_{n}\right) \in S, b_{i}>0, i=0, \ldots, n\right\}\right)}
$$

Denote by $T \subset \mathbb{Q}$ the semigroup generated by $\gamma(S)$. We have the following descriptions of $S$ and $T$ :

Lemma 3.2. (a) The semigroup $S$ is generated by $\left\{\mathbf{e}_{i}: i=0, \ldots, n\right\}$ and $S_{0}, \ldots, S_{n}$ where

$$
S_{i}:=\left\{\frac{1}{p_{i}}\left({\overline{k p_{0}}}^{p_{i}}, \ldots,{\overline{k p_{n}}}^{p_{i}}\right) \text { where }{\overline{k p_{n}}}^{p_{i}} \text { is the remainder in the division of } k p_{j} \text { by } p_{i}\right\}
$$

(b) $T$ is generated by $1 / \operatorname{lcm}\left(p_{i}, p_{j}\right), 0 \leq i<j \leq n$.

Proof. (a). Let $S^{\prime}$ be the semigroup generated by $\left\{\mathbf{e}_{i}: i=0, \ldots, n\right\}$ and $S_{0}, \ldots, S_{n}$. We want to show that $S=S^{\prime}$. Notice that $\widetilde{\alpha}\left(\mathbb{Z}^{n+1}\right)=\alpha(N)$ and by definition generates $S$. Let $\mathbf{a}=\left(a_{0}, \ldots, a_{n}\right) \in \mathbb{Z}^{n+1}$. We can assume without loss of generality that $a_{0} / p_{0}=$ $\min \left\{a_{i} / p_{i}: i=0, \ldots, n\right\}$. Let $u \in \mathbb{N}$ such that $k:=u p_{0}-a_{0} \in \mathbb{N}$. Writing the Euclidean division $k p_{i}=q_{i} p_{0}+\overline{k p}_{i} p_{0}$, we see that $a_{i}-\left(u p_{i}+q_{i}\right) \geq 0$. Then

$$
\widetilde{\alpha}(\mathbf{a})=\mathbf{a}-\frac{a_{0}}{p_{0}} \mathbf{p}=\left(0, a_{1}-\left(u p_{1}+q_{1}\right), \ldots, a_{n}-\left(u p_{n}+q_{n}\right)\right)+\frac{1}{p_{0}}\left(0,{\overline{k p_{1}}}^{p_{0}}, \ldots,{\overline{k p_{n}}}^{p_{0}}\right)
$$

from which it follows that $S \subset S^{\prime}$. For the reverse inclusion, it is enough to show that $S_{i} \subset S$. Again we show that $S_{0} \subset S$. Let $\frac{1}{p_{0}}\left(0,{\overline{k p_{1}}}^{p_{0}}, \ldots,{\overline{k p_{n}}}^{p_{0}}\right)$ be an element in $S_{0}$. There exists $q_{i} \in \mathbb{N}$ such that for $i \in\{0, \ldots, n\}$ we have

$$
-k p_{i}=-q_{i} p_{0}+{\overline{-k p_{i}}}^{p_{0}}=\left(1-q_{i}\right) p_{0}-{\overline{k p_{i}}}^{p_{0}} .
$$

Dividing by $p_{0} p_{i}$, we see that $-k / p_{0} \leq\left(1-q_{i}\right) / p_{i}$. We deduce that

$$
\alpha\left(-k, 1-q_{1}, \ldots, 1-q_{n}\right)=\frac{1}{p_{0}}\left(0,{\overline{k p_{1}}}^{p_{0}}, \ldots,{\overline{k p_{n}}}^{p_{0}}\right) .
$$


(b). As before we consider the semigroup $T^{\prime}$ generated by $1 / \operatorname{lcm}\left(p_{i}, p_{j}\right)$ for $0 \leq i<j \leq n$. In view of part (a), an element $\mathbf{s} \in S$ can be written as a finite sum

$$
\mathbf{s}=\sum_{i=0}^{n} s_{i} \mathbf{e}_{i}+\sum_{i=0}^{n} \mathbf{b}_{i}, s_{i} \geq 0
$$

with $\mathbf{b}_{i}$ in the semigroup generated by $S_{i}$. If we write $\mathbf{b}_{i}=\frac{1}{p_{i}}\left(b_{i, 0}, \ldots, b_{i, n}\right), \gamma(\mathbf{s})$ is given by

$$
\gamma(\mathbf{s})=\min \left\{\frac{1}{p_{j}}\left(s_{j}+\sum_{i=0}^{n} \frac{b_{i, j}}{p_{i}}\right): j=0, \ldots, n\right\}
$$

On the other hand, for any $0 \leq i \leq n$ there exists $k_{i} \in \mathbb{N}$ such that for any $j \in\{0, \ldots, n\}$ we have $b_{i, j} \equiv k_{i} p_{j} \bmod p_{i}$. In particular, $b_{i, i}=0$ and $b_{i, j} \in \operatorname{gcd}\left(p_{i}, p_{j}\right) \mathbb{Z}$ if $i \neq j$. As $\operatorname{lcm}\left(p_{i}, p_{j}\right) \operatorname{gcd}\left(p_{i}, p_{j}\right)=p_{i} p_{j}$, it follows that $\gamma(\mathbf{s}) \in T^{\prime}$. To see that $1 / \operatorname{lcm}\left(p_{i}, p_{j}\right) \in T$ for $i \neq j$, choose a positive integer $\ell$ such that $\ell p_{j} \equiv \operatorname{gcd}\left(p_{i}, p_{j}\right) \bmod p_{i}$. For $m \in\{0, \ldots, n\}$, set $\ell_{m} \equiv k p_{m} \bmod p_{i}$ with $0 \leq \ell_{m}<p_{m}$. Notice that $\ell_{j}=\operatorname{gcd}\left(p_{i} p_{j}\right)$. The element

$$
\mathbf{b}=\frac{1}{p_{i}}\left(\ell_{0}, \ldots, \ell_{n}\right)+\left(k_{0}, \ldots, \stackrel{j}{0}, \ldots, k_{n}\right)
$$

for $k_{m}$ sufficiently large, satisfies $\gamma(\mathbf{b})=1 / \operatorname{lcm}\left(p_{i}, p_{j}\right)$.

We are now ready to construct the fibration described in the introduction. Let $\ell=$ $1 / \operatorname{lcm}\left(p_{0}, \ldots, p_{n}\right)$ and $\bar{T}$ be the additive subgroup of $\mathbb{Q}$ generated by $\ell$. Let us also set $\ell_{i}=p_{i} \ell$ and denote by $\bar{S}$ the subgroup of $\mathbb{Q}^{n+1}$ generated by $\ell_{i} \mathbf{e}_{i}$. We have a well-defined commutative diagram respecting the addition:

$$
\begin{array}{cll}
T \underset{\uparrow}{\oplus} \stackrel{\phi^{*}}{\longrightarrow} \bar{S} & \phi^{*}(\gamma, \mathbf{b})=\mathbf{b}-\gamma \mathbf{p} \\
\pi_{\mathbf{p}}^{*} \uparrow_{T \underset{\psi^{*}}{\longrightarrow}} \bar{T} & \pi_{\mathbf{p}}^{*}(\gamma)=(\gamma, 0) \\
T & \rho^{*}(\lambda)=\lambda \mathbf{p} \\
&
\end{array}
$$

Notice that $\phi^{*}(\gamma, \mathbf{b})=0$ if and only if $(\gamma, \mathbf{b})=(\gamma(\mathbf{b}), \mathbf{b}-\alpha(\mathbf{b}))$. We denote by $t^{\gamma} \mathbf{z}^{\mathbf{b}}=$ $t^{\gamma} z_{0}^{b_{0}} \cdots z_{n}^{b_{n}}$ the corresponding element in $\mathbb{Q}[T \oplus S]$. Consider the ideal $I \subset \mathbb{Q}[T \oplus S]$ generated by

$$
\left\{\mathbf{z}^{\mathbf{b}}-t^{\gamma(\mathbf{b})} \mathbf{z}^{\alpha(\mathbf{b})}: \mathbf{b} \in S\right\}
$$

We obtain a commutative diagram of ring homomorphisms:

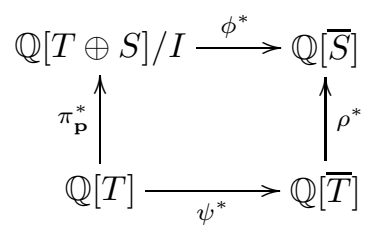

Consider now the elements $f_{\mathbf{w}}:=\sum_{i=0}^{n} \mathbf{z}^{\beta\left(\mathbf{e}_{i}\right)}=z_{0}^{w_{0}}+\cdots+z_{n}^{w_{n}} \in \mathbb{Q}[T \oplus S]$. Denote by $x_{i}$ the element $\mathbf{e}_{i}$ in $\mathbb{Q}[\bar{S}]$. We write $\mathbf{x}^{\mathbf{b}}=x_{0}^{b_{0}} \cdots x_{n}^{b_{n}}$ for the element $\mathbf{b} \in \mathbb{Q}[\bar{S}]$. Put $\bar{f}_{\mathbf{w}}=\sum_{i=0}^{n} \mathbf{x}^{\beta\left(\mathbf{e}_{i}\right)}=\sum_{i=0}^{n} x_{i}^{w_{i}} \in \mathbb{Q}[\bar{S}]$. Then, taking Spec of the diagram (27) we obtain the following. 
Theorem 3.3. The commutative diagram

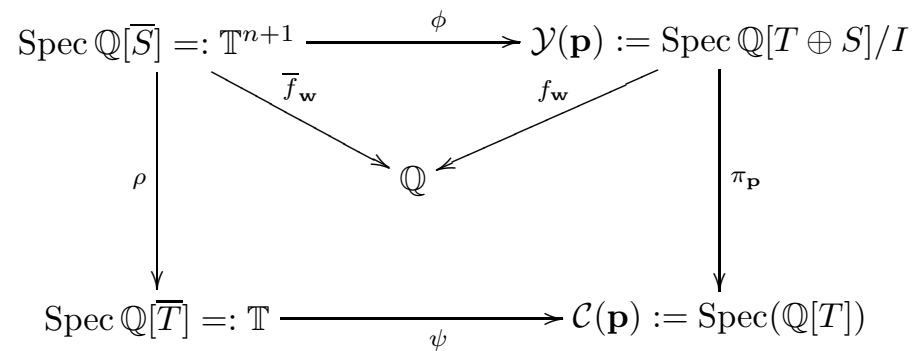

satisfies:

(a) $\phi$ and $\psi$ are isomorphism over their images;

(b) $\pi_{\mathbf{p}}$ is flat and

(c) the Jacobian algebra of $f$ over the 0 -fibre of $\pi_{\mathbf{p}}$ is isomorphic to the orbifold Chow ring $A_{\text {orb }}^{*}(\mathcal{X}(\mathbf{w}, \mathbf{p}))$.

Proof. The statements (a) and (b) are clear. For (c) we notice that $I+\left(\left\{t^{\gamma}: \gamma>0 \in T\right\}\right)$ is canonically isomorphic to the right hand side of (17) and hence isomorphic to $\mathbb{Q}[N]^{\Sigma}$. It remains to identify the module $\Theta_{\pi_{\mathbf{p}}}$ of $\mathbb{Q}[T]$-linear derivations of $\mathbb{Q}[T \oplus S]$ with the denominator of (8). According to Lemma 2.1] for any $\theta \in N^{\vee}$, the application

$$
\begin{aligned}
\xi_{\theta}: \mathbb{Q}[T \oplus S] & \longrightarrow \mathbb{Q}[T \oplus S] \\
t^{\gamma} \mathbf{z}^{\mathbf{b}} & \longmapsto \tilde{\theta}(\mathbf{b}) t^{\gamma} \mathbf{z}^{\mathbf{b}}
\end{aligned}
$$

is a derivation.

On the other hand $\xi_{\theta}(I) \subset I$ for $\tilde{\theta}(\mathbb{Q} \cdot \mathbf{p})=0$. Hence $\xi_{\theta}$ is a derivation of $\mathbb{Q}[T \oplus S] / I$ which is $\mathbb{Q}[T]$-linear by definition. We therefore obtain a map $\xi: N^{\vee} \rightarrow \Theta_{\pi_{\mathrm{p}}}, \theta \mapsto \xi_{\theta}$. To see that the image of $\xi$ freely generates $\Theta_{\pi_{\mathrm{p}}}$ over $\mathbb{Q}[T \oplus S] / I$ take $\theta_{1}, \ldots, \theta_{n}$ generators of $N^{\vee}$. Then $\xi_{\theta_{1}}, \ldots, \xi_{\theta_{n}}$ are independent over $\mathbb{Q}[T \oplus S] / I$ and, in view of the commutative diagram (28), we see that they generate the module $\Theta_{\mathcal{X}_{w(a)}}$ of derivations of the coordinate ring of the $\psi(q)$-fibre of $\pi_{\mathbf{p}}$. As no derivation can be supported only at the 0 -fibre of $\pi_{\mathbf{p}}$, we obtain the result.

Remark 3.4. C. Sabbah points out that the ring $\mathbb{Q}[N]^{\Sigma}$ can also be described as the graded algebra associated to the Newton filtration induced by $\beta$ on $N_{\mathbb{Q}}$. More precisely, let $\mathcal{P}$ be the convex hull of $\beta\left(\mathbf{e}_{i}\right)$ in $N_{\mathbb{Q}}$. It is a convex polyhedron containing the origin whose faces are defined by $L_{i}=1$, being $L_{i}$ the unique $\mathbb{Q}$-linear on $N_{\mathbb{Q}}$ with $L_{i}\left(\beta\left(\mathbf{e}_{j}\right)\right)=1$ for $i \neq j$. It thus defines the fan $\Sigma$. For $m \in N$, let us set $\nu(m):=\min \{\lambda \geq 0: m \in \lambda \cdot \mathcal{P}\}$ and define $\mathbb{Q}[N]_{\nu}$ as the vector space generated by $\mathbf{y}^{\mathbf{m}}$ with $\nu(\mathbf{m}) \leq \nu$. It is readily seen that the convexity of $\mathcal{P}$ implies that $\nu\left(\mathbf{m}+\mathbf{m}^{\prime}\right) \leq \nu(\mathbf{m})+\nu\left(\mathbf{m}^{\prime}\right)$ with equality if and only there exists a cone $\sigma \in \Sigma$ containing both $\mathbf{m}$ and $\mathbf{m}^{\prime}$. Hence we have

$$
\mathbb{Q}[N]^{\Sigma} \simeq \operatorname{gr}_{\mathcal{P}} \mathbb{Q}[N]=\bigoplus_{\nu \geq 0} \frac{\mathbb{Q}[N]_{\nu}}{\mathbb{Q}[N]_{<\nu}}
$$

In fact if we set $|\mathbf{b}|=\sum_{i=0}^{n} \frac{a_{i}}{w_{i}}$ for $\mathbf{b}=\left(b_{0}, \ldots, b_{n}\right) \in \mathbb{Q}^{n+1}$, it is easy to see that

$$
\nu\left(\mathbf{m}+\mathbf{m}^{\prime}\right)=|\alpha(\mathbf{m})|+\left|\alpha\left(\mathbf{m}^{\prime}\right)\right|-\left|\gamma\left(\alpha(\mathbf{m})+\alpha\left(\mathbf{m}^{\prime}\right)\right)\right|,
$$

and in particular, $\nu(\mathbf{m})=|\alpha(\mathbf{m})|$. This formula can be used to identify the fibration constructed in theorem 3.3 with a certain noetherian subring of $\oplus_{\nu \geq 0} \mathbb{Q}[N]_{\nu}$.

Remark 3.5. It is reasonable to expect some relation between the fibration (28) and the small quantum cohomology of weighted projective spaces as described in [CCLT06. 


\section{About the well-formed condition}

As we explain in the introduction after Theorem 1.1, we have not used that the weights $\mathbf{p}$ are well-formed. The proposition below justify this well-formed assumption.

Proposition 4.1. Let $(\mathbf{w}, \mathbf{p})$ and $\left(\mathbf{w}^{\prime}, \mathbf{p}^{\prime}\right)$ two pairs of weights such that $\operatorname{gcd}(\mathbf{p})=\operatorname{gcd}\left(\mathbf{p}^{\prime}\right)=$ 1. If the toric orbifolds $\mathcal{X}(\mathbf{w}, \mathbf{p})$ and $\mathcal{X}\left(\mathbf{w}^{\prime}, \mathbf{p}^{\prime}\right)$ are isomorphic then there exists two isomorphisms $g$ and $h$ such that the following diagram is commutative:

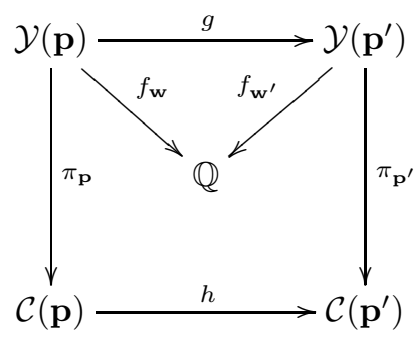

We start with a combinatorial Lemma.

Lemma 4.2. For any $i \in\{0, \ldots, n-1\}$ we have that for any $(j, k) \in\{0, \ldots, n-1\} \times \mathbb{N}$

$$
\frac{1}{p_{i}}{\overline{k p_{j}}}^{p_{i}}=\frac{1}{a p_{i}}{\overline{k a p_{j}}}^{a p_{i}}
$$

Proof. Without loss of generality, we can assume that $i=0$. There exists unique $\left(q,{\overline{k p_{j}}}^{p_{0}}\right) \in$ $\mathbb{N} \times\left\{0, \ldots, p_{0}-1\right\}$ and unique $\left(q^{\prime},{\overline{k a p_{j}}}^{p_{0}}\right) \in \mathbb{N} \times\left\{0, \ldots, a p_{0}-1\right\}$ such that $k p_{j}=q p_{0}+\overline{k p}_{j} p_{0}$ and $a k p_{j}=q^{\prime} a p_{0}+{\overline{k a p_{j}}}^{a p_{0}}$. By uniqueness, we deduce that $a q^{\prime}=q$ and $a \cdot{\overline{k p_{j}}}^{p_{0}}={\overline{k a p_{j}}}^{a p_{0}}$. This finishes the proof.

Proof of Proposition 4.1. According to the discussion at the end of Section 2 and Remark 2.3. it is enough to prove the proposition for the weights $\left(\mathbf{w}, a p_{0}, \ldots, a p_{n-1}, p_{n}\right)$ and $\left(w_{0}, \ldots, w_{n-1}, a w_{n}, \mathbf{p}\right)$ where $\operatorname{gcd}\left(p_{0}, \ldots, p_{n}\right)=1$ and $\operatorname{gcd}\left(a, p_{n}\right)=1$.

We will see that the isomorphism $g: \mathcal{Y}\left(a p_{0}, \ldots, a p_{n-1}, p_{n}\right) \rightarrow \mathcal{Y}\left(p_{0}, \ldots, p_{n}\right)$ sends $\left(z_{0}, \ldots, z_{n}\right)$ to $\left(z_{0}, \ldots, z_{n-1}, z_{n}^{1 / a}\right)$ and $h: \mathcal{C}\left(a p_{0}, \ldots, a p_{n-1}, p_{n}\right) \rightarrow \mathcal{C}\left(p_{0}, \ldots, p_{n}\right)$ sends $t$ to $t^{1 / a}$.

In our notation, we will stress for which family of weights we compute $S, T, \ldots$ We define a morphism of semigroups $h^{*}: T\left(p_{0}, \ldots, p_{n}\right) \rightarrow T\left(a p_{0}, \ldots, a p_{n-1}, p_{n}\right)$ that sends $\gamma$ to $\gamma / a$. For any $i, j \in\{0, \ldots, n-1\}$, we have $\operatorname{lcm}\left(a p_{i}, a p_{j}\right)=a \operatorname{lcm}\left(p_{i}, p_{j}\right)$ and for any $i \in$ $\{0, \ldots, n\}$, we have $\operatorname{lcm}\left(a p_{i}, p_{n}\right)=a \operatorname{lcm}\left(p_{i}, p_{n}\right)$. We deduce that $h: \mathcal{C}\left(a p_{0}, \ldots, a p_{n-1}, p_{n}\right) \rightarrow$ $\mathcal{C}\left(p_{0}, \ldots, p_{n}\right)$ is well-defined and is an isomorphism.

We define the morphism of semigroups :

$$
\begin{aligned}
\phi^{*}: S\left(p_{0}, \ldots, p_{n}\right) & \rightarrow S\left(a p_{0}, \ldots, a p_{n-1}, p_{n}\right) \\
\left(b_{0}, \ldots, b_{n}\right) & \mapsto\left(b_{0}, \ldots, b_{n-1}, \frac{b_{n}}{a}\right) .
\end{aligned}
$$

We will show that $\phi^{*}$ is an isomorphism. To prove that $\phi^{*}$ is well-defined, we show that:

(a) for $i \in\{0, \ldots, n\}$, we have $\phi^{*}\left(S_{i}\left(p_{0}, \ldots, p_{n}\right)\right) \subset S_{i}\left(a p_{0}, \ldots, a p_{n-1}, p_{n}\right)$

(b) and for $i \in\{0, \ldots, n\}$, we have $\phi^{*}\left(\mathbf{e}_{i}\right) \in S\left(a p_{0}, \ldots, a p_{n-1}, p_{n}\right)$.

(a). For $i=n$, it is obvious. For the case $i \neq n$, one can assume, without loss of generality, that $i=0$. Let $\frac{1}{p_{0}}\left(0,{\overline{k p_{1}}}^{p_{0}}, \ldots,{\overline{k p_{n}}}^{p_{0}}\right)$ be a generator of $S_{0}\left(p_{0}, \ldots, p_{n}\right)$. Let $u, v \in \mathbb{N}$ such that $u p_{n}-v a=1$. As we have that for any $t \in \mathbb{N}$

$$
\left(k+t u p_{0}\right) p_{n}=\left(\left\lfloor\frac{k p_{n}}{a p_{0}}\right\rfloor+v\right) a p_{0}+{\overline{k p_{n}}}^{a p_{0}}+t p_{0},
$$


we deduce that from there exists $t \in \mathbb{N}$ such that $0 \leq{\overline{\left(k+t u p_{0}\right) p_{n}}}^{a p_{0}} \leq p_{0}-1$. Putting $k^{\prime}:=k+t u p_{0}$, we have that ${\overline{k^{\prime} p_{n}}}^{a p_{0}}={\overline{k p_{n}}}^{p_{0}}$ and by Lemma 4.2 that for $j \in\{0, \ldots, n-1\}$ ${\overline{a k^{\prime} p_{j}}}^{a p_{0}}={\overline{a k p_{j}}}^{a p_{0}}$. We deduce that $\phi^{*}\left(S_{0}\left(p_{0}, \ldots, p_{n}\right)\right) \subset S_{0}\left(a p_{0}, \ldots, a p_{n-1}, p_{n}\right)$.

(b). For $i \in\{1, \ldots, n-1\}$, we have $\phi^{*}\left(\mathbf{e}_{i}\right) \in S\left(a p_{0}, \ldots, a p_{n-1}, p_{n}\right)$. For $i=n$, we put $k=u p_{0}$ where $u, v \in \mathbb{N}$ are the Bezout coefficients (i.e. $u p_{n}-v a=1$ ), we deduce that

$$
\phi^{*}\left(\mathbf{e}_{n}\right)=\frac{1}{a p_{0}}\left(0,{\overline{k a p_{1}}}^{a p_{0}}, \ldots,{\overline{k a p_{n-1}}}^{a p_{0}},{\overline{k p_{n}}}^{a p_{0}}\right) .
$$

We conclude that $\phi^{*}$ is well-defined.

The morhism $\phi^{*}$ is clearly injective and its image is contained in $S\left(a p_{0}, \ldots, a p_{n-1}, p_{n}\right)$. It hence suffices to show that it is also surjective. It is obvious that $S_{n}\left(a p_{0}, \ldots, a p_{n-1}, p_{n}\right) \subset$ $\operatorname{Im} \phi^{*}$. Let $\frac{1}{a p_{0}}\left(0,{\overline{k a p_{1}}}^{a p_{0}}, \ldots,{\overline{k a p_{n-1}}}^{a p_{0}},{\overline{k p_{n}}}^{a p_{0}}\right)$ be a generator of $S_{0}\left(a p_{0}, \ldots, a p_{n-1}, p_{n}\right)$ and consider the Euclidean division ${\overline{k p_{n}}}^{a p_{0}}=q p_{0}+{\overline{k p_{n}}}^{p_{0}}$ with $0 \leq{\overline{k p_{n}}}^{p_{0}}<p_{0}$, by Lemma 4.2. We deduce that

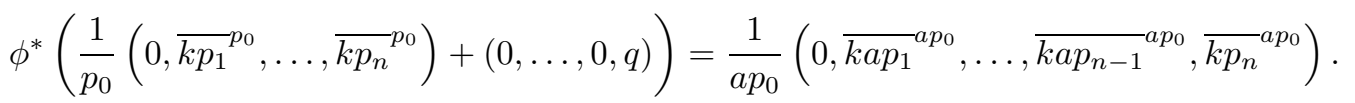

By the same argument, we deduce that $S_{j}\left(a p_{0}, \ldots, a p_{n-1}, p_{n}\right) \subset \operatorname{Im} \phi^{*}$. We conclude that $\phi^{*}$ is an isomorphism. We define the isomorphism of rings $\widetilde{g}^{*}:=\left(h^{*}, \phi^{*}\right): \mathbb{Q}[(T \oplus$ $\left.S)\left(p_{0}, \ldots, p_{n}\right)\right] \rightarrow \mathbb{Q}\left[(T \oplus S)\left(a p_{0}, \ldots, a p_{n-1}, p_{n}\right)\right]$.

As for any $b \in S$ we have $h^{*}(\gamma(b))=\gamma\left(\phi^{*}(b)\right)$ and $\phi^{*}(\alpha(b))=\alpha\left(\phi^{*}(b)\right)$, we deduce that $\widetilde{g}^{*}$ induces an isomorphism of rings

$$
g^{*}: \mathbb{Q}\left[(T \oplus S)\left(p_{0}, \ldots, p_{n}\right)\right] / I\left(p_{0}, \ldots, p_{n}\right) \rightarrow \mathbb{Q}\left[(T \oplus S)\left(p_{0}, \ldots, p_{n}\right)\right] / I\left(a p_{0}, \ldots, a p_{n-1}, p_{n}\right)
$$

The induced morphism of schemes $g: \mathcal{Y}\left(a p_{0}, \ldots, a p_{n-1}, p_{n}\right) \rightarrow \mathcal{Y}\left(p_{0}, \ldots, p_{n}\right)$ sends $\left(z_{0}, \ldots, z_{n}\right)$ to $\left(z_{0}, \ldots, z_{n-1}, z_{n}^{1 / a}\right)$ is an isomorphism such that the diagram of Proposition 4.1 is commutative.

\section{Examples}

In this section we have used Singular GPS05]) to embed the fibration (28) into affine spaces. We illustrated for two different cases: $\mathbf{p}=(2,3,5)$ and $\mathbf{p}=\left(p_{0}, \ldots, p_{n}\right)$ where $p_{0}=1$ and $p_{i}$ divides $p_{i+1}$. We begin with the latter.

Case $\mathbb{P}(\mathbf{p})$ with $p_{i} \mid p_{i+1}$. Set $p_{i}=d_{i} p_{i-1}$. Let $v_{i}=z_{0}^{p_{0} / p_{i}} \ldots z_{i-1}^{p_{i-1} / p_{i}}$ for $i=1, \ldots, n$. The monomials $v_{i}$ correspond to the generators of $S$ described in [3.2, (a). We thus have the relations $v_{i+1}^{d_{i+1}}-v_{i} z_{i}, i=1, \ldots, n-1$ and it is easy to see that in fact these generate all the relations between the monomials in $v_{i}$ and $z_{i}$. On the other hand, we have $\mathbb{Q}[T]=\mathbb{Q}\left[t^{1 / p_{n}}\right] \simeq$ $\mathbb{Q}[s]$ and the ideal $I \in \mathbb{Q}[T \oplus S]$ is generated by one single element, namely $v_{n} z_{n}-s=0$. Then

$$
\mathcal{X}=\left\{v_{1}^{d_{1}}=z_{0}, v_{2}^{d_{2}}=v_{1} z_{1}, v_{3}^{d_{3}}=v_{2} z_{2}, \ldots, v_{n}^{d_{n}}=v_{n-1} z_{n-1}, v_{n} z_{n}=s\right\} \hookrightarrow \mathbb{A}^{2 n+2}
$$

and $\pi: \mathcal{X} \rightarrow \mathbb{A}^{1}$ is the restriction of the projection onto the $s$-line.

Case $\mathbb{P}(2,3,5)$. To describe $\mathbb{Q}[S]$ for the case $\mathbf{p}=(2,3,5)$, consider the monomials

$$
w_{1}=z_{0}^{2 / 5} z_{1}^{3 / 5}, w_{2}=z_{0}^{4 / 5} z_{1}^{1 / 5}, w_{3}=z_{0}^{1 / 5} z_{1}^{4 / 5}, w_{4}=z_{0}^{3 / 5} z_{1}^{2 / 5}
$$

with relations

$$
w_{1}^{2}=w_{2} z_{1}, w_{1} w_{2}=w_{3} z_{0}, w_{1} w_{3}=w_{4} z_{1}, w_{1} w_{4}=z_{0} z_{1}
$$


Similarly, we have monomials $u_{1}$ and $v_{1}$ corresponding to the elements with denominator $1 / 2$ and $1 / 3$ with relations

$$
u_{1}^{2}=z_{1} z_{2}, v_{1}^{3}=z_{0} z_{3} .
$$

On the other hand we have $\mathbb{Q}[T]=\mathbb{Q}\left[t^{1 / 6}, t^{1 / 10}, t^{1 / 15}\right]$ so that we have the embedding $\mathcal{C} \hookrightarrow \mathbb{A}^{3}$ as the rational curve

$$
s_{1}=s_{2} s_{3}, s_{2}^{3}=s_{1} s_{3}^{2}, s_{3}^{3}=s_{2}^{2}
$$

Finally, the ideal $I$ is given by

$$
\begin{gathered}
u_{1} v_{1}=s_{1} \\
u_{1} w_{1}=s_{2} w_{2}, u_{1} w_{2}=s_{2} w_{4}, u_{1} w_{3}=s_{2} z_{1}, u_{1} w_{4}=s_{2} w_{1} \\
v_{1} w_{1}=s_{3} w_{4}, v_{1} w_{2}=s_{3} z_{0}, v_{1} w_{3}=s_{3} w_{1}, v_{1} w_{4}=s_{3} w_{2}
\end{gathered}
$$

Therefore $\mathcal{X} \hookrightarrow \mathbb{A}^{12}$ is defined by the equations (40), (41), (42) and (43), with the fibration $\pi: \mathcal{X} \rightarrow \mathcal{C}$ given by the projection onto the $\left(s_{1}, s_{2}, s_{3}\right)$-space.

Notice that in any of the above cases we can obtain presentations of $A_{\text {orb }}^{*}(\mathcal{X}(\mathbf{w}, \mathbf{p}))$ by setting $s=0$ and adding the equations

$$
\frac{w_{i}}{p_{i}} z_{i}^{w_{i}}-\frac{w_{i+1}}{p_{i+1}} z_{i}^{w_{i+1}}, i=0, \ldots, n-1 .
$$

Acknowledments. The first author is grateful to B. Dubrovin and B. Fantechi for making it possible to visit the Scuola Internazionale Superiore di Studi Avanzati where this article was started.

\section{References}

[AGV02] Dan Abramovich, Tom Graber, and Angelo Vistoli, Algebraic orbifold quantum products, Orbifolds in mathematics and physics (Madison, WI, 2001), Contemp. Math., Amer. Math. Soc., Providence, RI, 2002, pp. 1-24.

[Bar00] Serguei Barannikov, Semi-infinite Hodge structures and mirror symmetry for projective spaces, arXiv.math.AG/0010157 (2000), 17.

[BCS05] Lev A. Borisov, Linda Chen, and Gregory G. Smith, The orbifold Chow ring of toric Deligne-Mumford stacks, J. Amer. Math. Soc. (2005), no. 1, 193-215 (electronic).

[CCLT06] Tom Coates, Alessio Corti, Yuan-Pin Lee, and Hsian-Hua Tseng, The quantum orbifold cohomology of weighted projective space, math.AG/0608481 (2006).

[CR04] Weimin Chen and Yongbin Ruan, A new cohomology theory of orbifold, Comm. Math. Phys. (2004), no. 1, 1-31.

[dG06] Ignacio de Gregorio, Deformations of functions and F-manifolds, Bull. London Math. Soc. 38 (2006), no. 6, 966-978.

[FMN07] Barbara Fantechi, Etienne Mann, and Fabio Nironi, Smooth toric dm stacks, arXiv.AG:0708.1254 (2007).

[Giv98] Alexander Givental, A mirror theorem for toric complete intersections, Topological field theory, primitive forms and related topics (Kyoto, 1996), Progr. Math., vol. 160, Birkhäuser Boston, Boston, MA, 1998, pp. 141-175.

[GPS05] G.-M. Greuel, G. Pfister, and H. Schönemann. Singular 3.0. A Computer Algebra System for Polynomial Computations. Centre for Computer Algebra, University of Kaiserslautern (2005). http://www.singular.uni-kl.de.

[Man06] Etienne Mann, Orbifold quantum cohomology of weighted projective spaces, arXiv.math.AG/0610965 (2006). 
Ignacio de Gregorio

Mathematics Institute

University of Warwick

CV4 7AL, Coventry

United Kingdom

Tel.: +442476 524 402

I.de-Gregorio@warwick.ac.uk
Étienne Mann

Institut de Mathématiques et Modélisation

Université Montpellier 2

Case Courrier 051

Place Eugéne Bataillon

34095 Montpellier Cedex

France

emann@math . univ-montp2. fr 